\title{
Editorial: Fun with Algorithms
}

\author{
Evangelos Kranakis • Danny Krizanc • \\ Flaminia Luccio
}

Published online: 20 September 2013

(C) Springer Science+Business Media New York 2013

... I simply enjoy giving more than receiving in every respect, do not take myself nor the doings of the masses seriously, am not ashamed of my weaknesses and vices, and naturally take things as they come with equanimity and humor.

A. Einstein to M. Born

April 12, 1949,

Einstein Archives 8-223.

What really makes algorithmic mathematics fun? One might claim, "it is the pleasure of the pure mathematical discovery brought by simple curiosity". Another would assert, "implementing an algorithm in a computer so as to find an unexpected connection to mathematical reasoning". Be what may, the truth is that algorithmics research can be fun, when done by people who have fun doing it, and who take things as they come... with humor! No doubt, it is not so easy to convey why fun with algorithms is fun!

E. Kranakis (凶)

School of Computer Science, Carleton University, 1125 Col. By Dr., Ottawa, ON K1S 5B6, Canada e-mail:kranakis@scs.carleton.ca

D. Krizanc

Mathematics Department, Wesleyan University, Middletown, CT 06459, USA

e-mail: dkrizanc@wesleyan.edu

F. Luccio

Dipartimento di Scienze Ambientali, Statistica e Informatica, Università Ca’ Foscari Venezia, via Torino 155, 30172, Venezia Mestre (Ve), Italy

e-mail: luccio@unive.it 
This issue contains a variety of papers with a multitude of interesting methodologies and approaches all brought together in the name of fun. It is of course hard to do justice to the elegant, rich ideas presented in the papers or even give details of the research work in such a short introduction, but a brief outline of the papers presented is as follows.

Picture-Hanging Puzzles shows how to hang a picture by wrapping rope around $n$ nails, making a polynomial number of twists, such that the picture falls whenever any $k$ out of the $n$ nails get removed, and the picture remains hanging when fewer than $k$ nails get removed. The Coolest Way to Generate Binary Strings gives interesting ways of generating all binary strings, or weight-range binary strings by providing generalized successor rules and the orders can be generated by simple algorithms. Perfect Strategies for the Ulam-Renyi Game with Multi-Interval Questions studies a new variant of the classical 20 question game with lies having the additional constraint on the questions that the subsets they ask should be the union of at most $k$ intervals for some fixed $k>0$. Gaming is a Hard Job, but Someone Has To Do It! establishes some general schemes relating the computational complexity of a video game to the presence of certain common elements or mechanics and applies them to the analysis of several video games such as Pac-Man, Tron, Lode Runner, etc. Optimal Sensor Networks for Area Monitoring using Rotating and Beam Sensors considers the problem of monitoring the Euclidean plane using rotating sensors with detection sectors and beam sensors assuming that intruders can appear anywhere at any time and move arbitrarily fast, and may have full knowledge of the sensor network. Computational Aspects of M.C. Escher's Ribbon Patterns is concerned with nice combinatorial questions arising from the beautiful and aesthetic artwork of Escher's designs. Practical Algorithms for Generating a Random Ordering of the Elements of a Weighted Set discusses the problem of efficiently generating a random ordering of the elements of an $n$-element weighted set, where the elements' weights are interpreted as relative probabilities. Scandinavian Thins on Top of Cake: New and Improved Algorithms for Stacking and Packing shows how to compute the smallest rectangle that can enclose any polygon, from a given set of polygons, in nearly linear time and also gives efficient algorithms for other similar enclosure problems. The Kissing Problem: How to End a Gathering When Everyone Kisses Everyone Else Goodbye introduces the kissing problem: given a rectangular room with $n$ people in it, what is the most efficient way for each pair of people to kiss each other goodbye? Spanning Trees and the Complexity of Flood-Filling Games is related to the combinatorial game (Free-) Flood-It, in which players aim to make a coloured graph monochromatic with the minimum possible number of flooding operations.

First and foremost we would like to thank the authors that contributed to this issue. We would like to express our deepest gratitude to all the (anonymous) reviewers, who carefully worked out all the details in the papers and provided insightful comments and remarks to the authors. Without them this collection of papers would have never been possible. Finally, we wish to thank the competent staff at Springer for facilitating our work. We hope you will enjoy the papers as much as we did.

August 12, 2013 